${ }^{2}$ Mc Gill University, Rheumatology, Montreal, Canada; ${ }^{3}$ Western University, Rheumatology, London, Canada

Background: Patients with Raynaud's Phenomenon (RP) from systemic sclerosis (SSc) may experience severe complications. Digital ulcers (DUs), occur in approximately half the patients with SSc, and cause hand dysfunction, severe pain, and decreased quality of life. DUs lead to increased healthcare utilization and systemic economic burden through hospitalizations, ED visits, and ambulatory services (1). However, access to medications such as PDE5 inhibitors and prostacyclins that are within the EULAR/EUSTAR SSc guidelines (2) in a country with global health care but patchy pharmacare such as Canada has not been studied.

Objectives: The purpose of this study was to elucidate the access to treatment of medications for RP and DU in patients with SSc in Canadian provinces through identifying the provincial and private insurance coverage of PDE5 inhibitors (PDE5i) and prostanoids, the timelines and procedures of requesting these medications, and the process of administering IV prostanoids if required for patient care.

Methods: We designed an online survey and collected data through the Survey Monkey platform. The survey was administered to rheumatologists affiliated with the Canadian Scleroderma Research Group (CSRG) from December 2020 to January 2021. Responders were asked to report if the province or private insurance automatically provided PDE5i for patients with RP and DU or if a dedicated process was required to attain these medications. Additionally, responders were asked to describe the process of administering lloprost, Epoprostenol and Alprostadil and the barriers inherent to their administration. Of note, there is no DIN number for lloprost in Canada so every time it is used there must be an application to Health Canada.

Results: The survey was completed by $100 \%$ of CSRG researchers (17/17), representing 8 provinces in Canada. None of the provincial governments provided coverage for PDE5i without special requests that were adjudicated on a case by case basis with approximately half the provinces paying for PDE5i upon special request if a patient was eligible for provincial drug insurance (ex elderly, youths, low income families). Two provinces, Quebec and Saskatchewan, provided PDE5i "all the time". Whereas NS, MB, ON, BC, and AB provided them "sometimes"; NFLD provided them "never". Provincial governments and private insurance fulfilled requests "within 1 month" $62 \%$ of the time and the other requests took longer to be answered. Private insurance approved coverage with special request in $\mathrm{AB}, \mathrm{MB}, \mathrm{QC}, \mathrm{ON}$, and NS. Respondents described administration of IV prostanoids as "inconsistent", requiring "a lot of work", and that patients in most jurisdictions be admitted as in-patients for provinces to cover these medications. Conclusion: Most jurisdiction within Canada do not provide coverage for PDE5i and the process to obtain access for patients is delayed, non-uniform, and often not approved. Intravenous prostanoid infusions are difficult to obtain and have system barriers. Advocacy and cost effectiveness data should be used to advocate for access to medications that are recommended within SSC recommendations.

REFERENCES:

[1] Morrisroe K, et al. Digital ulcers in systemic sclerosis: their epidemiology, clinical characteristics, and associated clinical and economic burden. Arthritis research \& therapy. 2019 Dec;21(1):1-2.

[2] Kowal-Bielecka O, et al. Update of EULAR recommendations for the treatment of systemic sclerosis. Annals of the rheumatic diseases. 2017 Aug 1:76(8):1327-39.

Disclosure of Interests: None declared

DOI: 10.1136/annrheumdis-2021-eular.1265

\section{AB0420 \\ CIRCULATING MONOCYTES HAVE DISTINCT PHYSICAL PROPERTIES THAT CORRELATE WITH DISEASE ACTIVITY AND SEVERITY AND PREDICT PROGRESSION IN SYSTEMIC SCLEROSIS}

A. E. Matei ${ }^{1}$, K. Markéta ${ }^{2}$, A. H. Györfi ${ }^{1}$, E. Boxberger ${ }^{1}$, D. Soteriou ${ }^{2}$ M. Papava ${ }^{1}$, J. Muth ${ }^{1}$, M. Kräter ${ }^{2,3}$, G. Schett ${ }^{1}$, J. Guck ${ }^{2,3}$, J. H. W. Distler ${ }^{1}$ ${ }^{1}$ Friedrich-Alexander-University Erlangen-Nürnberg (FAU) and University Hospital Erlangen, Department of Internal Medicine 3 - Rheumatology and Immunology; 2Deutsches Zentrum für Immuntherapie (DZI), Erlangen, Germany; ${ }^{2}$ Max Planck Institute for the Science of Light \& Max-PlanckZentrum für Physik und Medizin, Guck Division, Erlangen, Germany; ${ }^{3}$ Center for Molecular and Cellular Bioengineering, Technische Universität Dresden, Biotechnology Center, Dresden, Germany
Background: Systemic sclerosis (SSc) is associated with high morbidity and is one of the autoimmune rheumatic diseases with the highest mortality. However, tools to evaluate disease activity, response to treatment or to predict disease progression are scarce. Dysregulated immune responses are major pathogenic players at the onset and in the progression of SSc. Recent evidence demonstrates that mechanical properties of circulating leukocytes reflect their states and functions, and during activation ensure their adaptation to the changing physical requirements (e.g. softening to extravasate and migrate in the tissues) (1). Real-time fluorescence and deformability cytometry (RT-FDC) is a novel technique that allows the identification of cells from a heterogenous population by marker expression, with their subsequent mechanical phenotyping in a high-throughput manner $(2,3)$.

Objectives: Here we characterized the physical properties of circulating immune cells in SSc patients, aiming to identify disease-related changes in their phenotypes, clinical correlates of these changes and their potential to predict disease progression.

Methods: 51 patients fulfilling the 2013 ACR/EULAR classification criteria for SSc and 17 age- and sex-matched healthy controls were included in the study. Blood was collected from the donors between 05.2019 and 10.2020. Peripheral blood mononuclear cells (PBMCs) were isolated and stained with antibodies against major circulating lymphoid $\left(\mathrm{CD}^{+}, \mathrm{CD}^{+} \mathrm{T}\right.$ cells, B cells, NK cells, NKTlike cells) and myeloid subpopulations (classical, intermediate and inflammatory monocytes, conventional dendritic cells and plasmacytoid dendritic cells). Each subpopulation was identified in RT-FDC by standard gating based on its marker expression and its area, deformation and apparent Young's modulus (a measure of cell stiffness) were determined. The analysis was conducted using a custom Python script. For the patients included, demographic and clinica data were collected at every visit. Correlations with clinical parameters were analyzed in R.

Results: All three subpopulations of monocytes identified by expression of HLA-DR, CD14 and/or CD16 had higher deformation and cross-sectional area in SSc patients as compared to healthy controls. From the SSc patients, monocytes had higher deformation and area in those with diffuse cutaneous SSc extensive lung fibrosis and active disease as compared to those with limited cutaneous SSc, limited lung fibrosis and stable disease, respectively. Moreover monocyte deformation and area significantly correlated with the EUSTAR activity index, with mRSS, with the extent of lung involvement on HR-CT (positive correlation), with DLCO and FVC (negative correlation). Follow-up data collected one year after the measurements showed that a higher monocyte deformation and cross-sectional area at baseline predicts future progression of lung disease, defined according to the INBUILD study, as well as future progression of skin fibrosis.

Conclusion: We demonstrated that circulating subsets of monocytes in SSC patients show an increase in deformation and cross-sectional area, that these changes correlate with current disease activity and can identify patients with high risk of future progression of skin or lung fibrosis. These changes might reflect an activated state of circulating monocytes in SSc that facilitate their tissue migration. Mechanical phenotyping of monocytes by RT-FDC might thus serve as a useful tool for clinical evaluation of SSc patients.

\section{REFERENCES:}

[1] Bashant KR, Toepfner N, Day CJ, Mehta NN, Kaplan MJ, Summers C, et al. The mechanics of myeloid cells. Biol Cell. 2020;112(4):103-12

[2] Otto O, Rosendahl P, Mietke A, Golfier S, Herold C, Klaue D, et al. Real-time deformability cytometry: on-the-fly cell mechanical phenotyping. Nat Methods. 2015;12(3):199-202, 4 p following

[3] Rosendahl P, Plak K, Jacobi A, Kraeter M, Toepfner N, Otto O, et al. Realtime fluorescence and deformability cytometry. Nat Methods. 2018;15(5): 355-8.

Disclosure of Interests: Alexandru-Emil Matei: None declared, Kubánková Markéta: None declared, Andrea-Hermina Györfi: None declared, Evgenia Boxberger: None declared, Despina Soteriou: None declared, Maria Papava: None declared, Julia Muth: None declared, Martin Kräter: None declared, Georg Schett: None declared, Jochen Guck: None declared, Jörg H.W. Distler Consultant of Actelion, Active Biotech, Anamar, ARXX, Bayer Pharma, Boehringer Ingelheim Celgene, Galapagos, GSK, Inventiva, JB Therapeutics, Medac, Pfizer, RuiYi and UCB, Grant/research support from: Anamar, Active Biotech, Array Biopharma, aTyr, BMS, Bayer Pharma, Boehringer Ingelheim, Celgene, Galapagos, GSK, Inventiva, Novartis, Sanofi-Aventis, RedX, UCB, Employee of: Stock owner of 4D Science and Scientific head of FibroCure DOI: 10.1136/annrheumdis-2021-eular.1274 